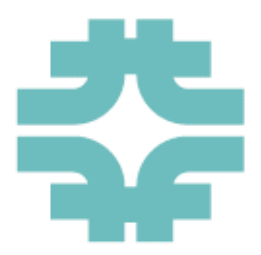

Superconducting R\&D

Magnet System Department

Fermilab's Technical Division

\title{
Analytical Study of Stress State in HTS Solenoids
}

Author:

Supervisor:
Emanuele Terzini

Emanuela Barzi 


\section{INDEX}

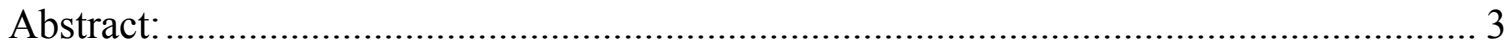

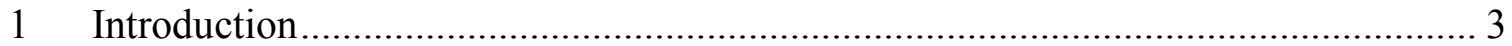

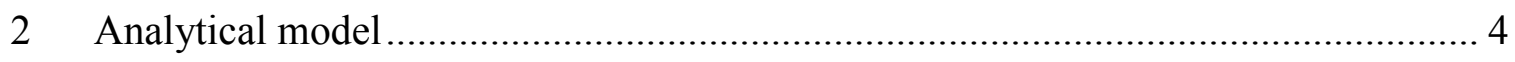

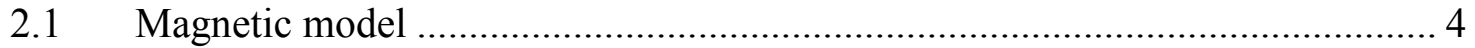

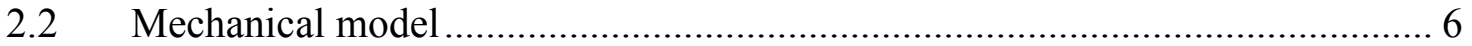

2.3 Stress state for several constraints hypotheses............................................. 7

2.4 Stress state as a function of Self-Field ....................................................... 9

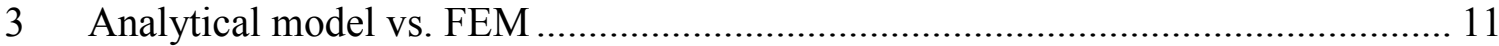

4 Effect of temperature and of finite rigidity of the constraints (BSCCO vs. YBCO



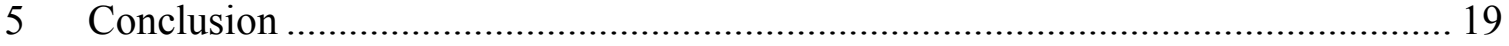






\section{Abstract:}

A main challenge for high field solenoids made of in High Temperature Superconductor (HTS) is the large stress developed in the conductor. This is especially constraining for BSCCO, a brittle and strain sensitive ceramic material. To find parametric correlations useful in magnet design, analytical models can be used. A simple model is herein proposed to obtain the radial, azimuthal and axial stresses in a solenoid as a function of size, i.e. self-field, and of the engineering current density for a number of different constraint hypotheses. The analytical model was verified against finite element modeling (FEM) using the same hypotheses of infinite rigidity of the constraints and room temperature properties. FEM was used to separately evaluate the effect of thermal contractions at $4.2 \mathrm{~K}$ for $\mathrm{BSCCO}$ and $\mathrm{YBCO}$ coils. Even though the analytical model allows for a finite stiffness of the constraints, it was run using infinite stiffness. For this reason, FEM was again used to determine how much stresses change when considering an outer stainless steel skin with finite rigidity for both BSCCO and YBCO coils.

\section{Introduction}

For a better understanding of the actual loads that high field solenoids made of HTS will be subject to, we have started some analytical studies of stress state in solenoids for a number of constraint hypotheses. This will hopefully show what can be achieved with the present conductor in terms of self-field. The magnetic field $(B)$ exerts a force $F=B \times J$ per unit volume. In superconducting magnets, where the field and current density $(J)$ are both high, this force can be very large, and it is therefore important to calculate the stresses in the coil.

To produces the stresses as a function of a coil nominal field, the following was done:

- Analytical magnetic model.

- Analytical mechanical model:

* Solution for unconstrained coil;

* Solutions for a number of constraint hypotheses.

Of the several variables that define the problem, the inner diameter of the solenoid was set to $50 \mathrm{~mm}$. In a first stage of this study, the critical current density in the 
superconductor $\left(J_{c}\right)$ was taken equal to $244 \mathrm{~A} / \mathrm{mm}^{2}$, i.e. the extrapolated $J_{c}$ value at $\sim 40 \mathrm{~T}$ [1]. All other properties are taken at room temperature. The effect of temperature was calculated separately for BSCCO and for YBCO coils using finite element modeling (FEM) in Section 4.

The engineering current density $J$ was calculated taking into account voids between the $0.8 \mathrm{~mm}$ (d) round strands, and $0.05 \mathrm{~mm}(\mathrm{t})$ of insulation around each strand, as in Fig. 1, yielding:

$$
J=\frac{J_{c} \frac{\pi}{4} d^{2}}{(d+2 t)^{2}}=151.6 \quad \frac{\mathrm{A}}{\mathrm{mm}^{2}}
$$

\section{Analytical model}

Fig. 1. Strand configuration in coil model.

\subsection{Magnetic model}

Our solenoid is shown in Fig. 2. In the case of a length $L=\infty$, it is straightforward to estimate the magnetic field at the center of the solenoid from the following equation [2]:

$$
B_{\infty}(\alpha)=R 1(\alpha-1) \mu_{0} J
$$

where:

- $\alpha$ is the ratio of outer radius (R2) over inner radius $(R I=0.025 \mathrm{~m})$

- $\mu_{0}$ is the magnetic permeability constant equal

$$
\text { to } 4 \pi 10^{-7} \mathrm{~T} \mathrm{~m} / \mathrm{A}
$$

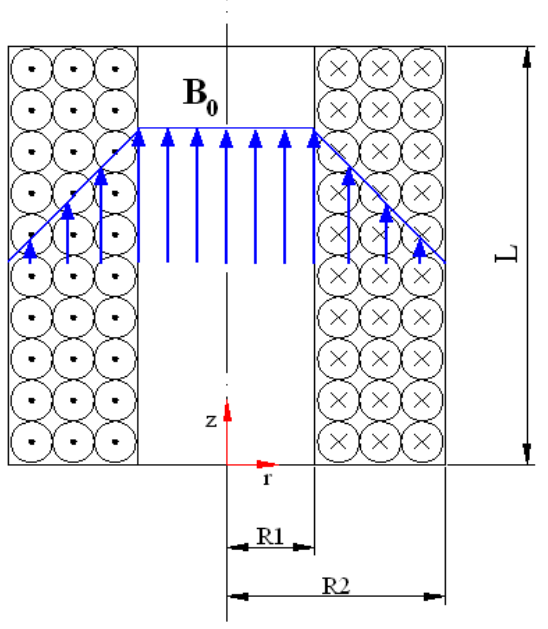

Fig. 2. Solenoid model.

- $J$ is the engineering current density in $A / \mathrm{m}^{2}$

In Fig. 2 we use also the hypothesis of a linear trend of the magnetic field inside the solenoid. A more realistic calculation can be made for a solenoid with finite length; in this case the magnetic field can be estimated from the following equation [3]: 
$B_{0}(\alpha, \beta)=R 1 \mu_{0} J \beta \ln \left(\frac{\sqrt{\alpha^{2}+\beta^{2}}+\alpha}{\sqrt{1+\beta^{2}}+1}\right)$

where $\beta=(L / 2) / R l$ is the solenoid dimensionless length with respect to the inner radius.

In Fig. 3 equations (1) and (2) are plotted for solenoids of various lengths. In the legend of the plot in Fig. 3 (left), $\mathrm{B}_{0}$ is expressed as a function of $\beta$. In the legend of the plot in Fig. 3 (right), $\mathrm{B}_{0}$ is expressed as a function of $L(\mathrm{~m})$.

To represent the stress state along the thickness of the solenoid, $\alpha$ was taken equal to 4 , and the length of the solenoid was chosen by minimizing the volume using the plot shown in Fig. 4 [4]. In our case we have the minimum volume of the solenoid for $\beta=2.52$ and therefore a length of the solenoid equal to $126 \mathrm{~mm}$. Fig. 5 shows the characteristic of the solenoid in question.
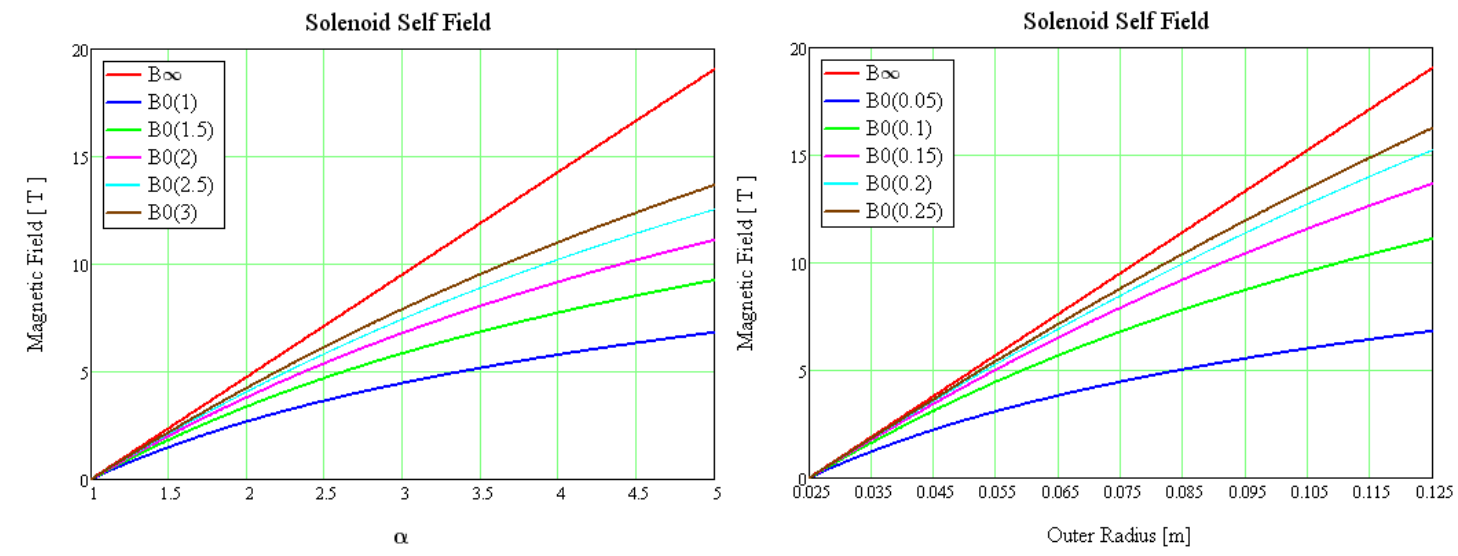

Fig. 3. Self-field vs. outer size for solenoids of various lengths.



Fig. 4. Minimum Volume curve.

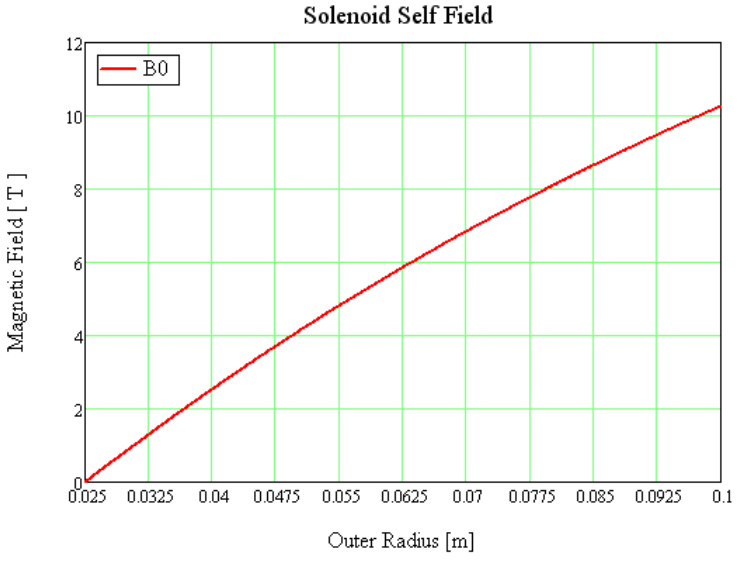

Fig. 5. Characteristic of the solenoid. 
Summarizing, we have:

- Inner diameter $=50 \mathrm{~mm}$

- Outer diameter $=200 \mathrm{~mm}$

- $\quad$ Length $=126 \mathrm{~mm}$

- $\mathrm{B}_{0}=10.26 \mathrm{~T}$

\subsection{Mechanical model}

The mechanical model is based on the assumptions that the coil elastic properties are isotropic, to solve the problem analytically using the standard techniques for distributed forces in a cylinder [5].

Referencing to Fig. 6 where the $\mathrm{z}$ axis is parallel to the solenoid axis (shear stresses are assumed negligible), by using the local displacement in the radial direction $(u)$ as our working variable we may reduce the condition for equilibrium between radial stress

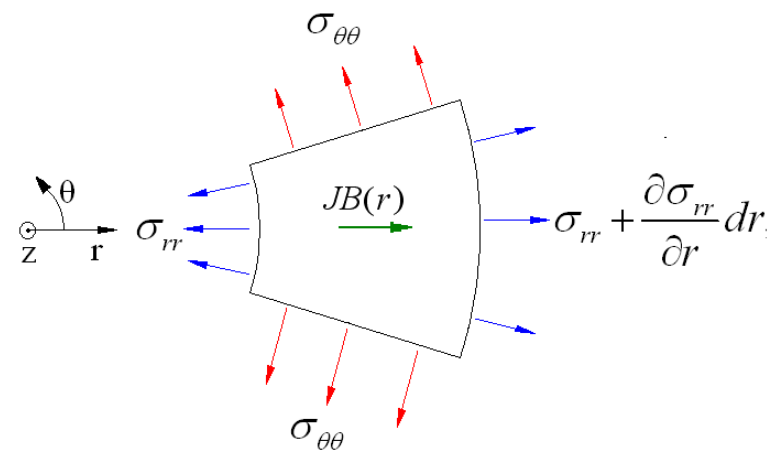

Fig. 6. Infinitesimal element. $\left(\sigma_{r r}\right)$, hoop stress $\left(\sigma_{\theta \theta}\right)$ and magnetic load $(B J)$ to a single equation (Lame's equation):

$$
\frac{d}{d r}\left(\frac{1}{r}\left(\frac{d}{d r}(r \cdot u)\right)\right)=-\frac{\left(1-v^{2}\right)}{E} J B(r)
$$

where:

- $\quad v$ is the Poisson's modulus [-]

- $E$ is the Young's modulus $\left[\mathrm{N} / \mathrm{m}^{2}\right]$

Once that the solution of (3) is found, we can estimate the circumferential $\left(\varepsilon_{\theta \theta}\right)$ and radial $\left(\varepsilon_{r r}\right)$ strain and then the hoop and radial stress according to:

$$
\begin{aligned}
& \varepsilon_{\theta \theta}(r)=\frac{u}{r}, \varepsilon_{r r}(r)=\frac{d u}{d r} \\
& \sigma_{\theta \theta}(r)=\frac{E}{1-v^{2}}\left(\varepsilon_{\theta \theta}(r)+v \varepsilon_{r r}(r)\right), \sigma_{r r}(r)=\frac{E}{1-v^{2}}\left(\varepsilon_{r r}(r)+v \varepsilon_{\theta \theta}(r)\right)
\end{aligned}
$$


The generalized plain strain theory allows calculating the axial stress as follows:

$$
\sigma_{z z}(r)=v\left(\sigma_{\theta \theta}(r)+\sigma_{r r}(r)\right)-\overline{\sigma_{z z}}
$$

where:

$$
\overline{\sigma_{z z}}=\frac{2 \pi \int_{R_{1}}^{R_{2}} \sigma_{z z}(r) \cdot r \cdot d r}{\pi\left(R_{2}^{2}-R_{1}^{2}\right)} \text { is the average axial stress. }
$$

All the stresses calculated above are in the middle section on the solenoid, i.e. at $\mathrm{z}=\mathrm{L} / 2$.

\subsection{Stress state for several constraints hypotheses}

The five constraints hypotheses illustrated in Fig. 7 have been modeled analytically with infinite rigidity. The effect of a finite rigidity was calculated using FEM in Section 4 for both BSCCO and YBCO coils.

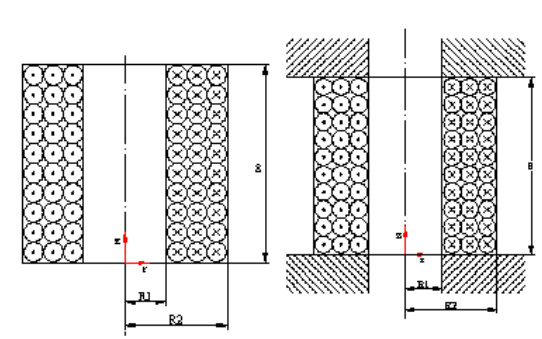

a)

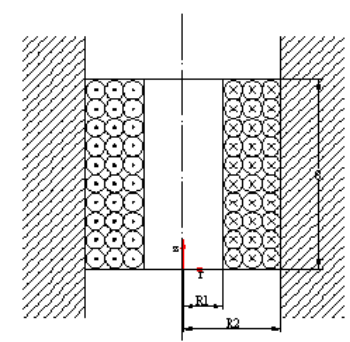

c)

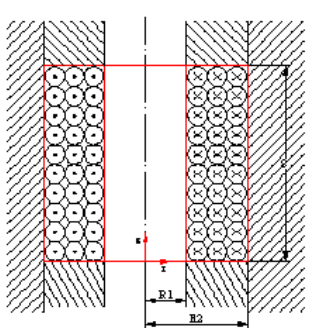

d)

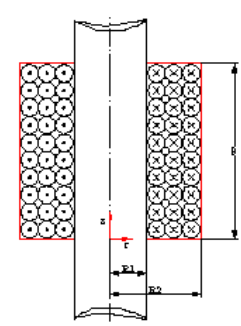

e)

Fig. 7. Constraint hypotheses: a) unconstrained solenoid; b) Upper/Lower surface Locked; c) Outer surface locked; b) and c) together; e) Inner surface locked.

The boundary conditions for each configuration are:

- a) $\sigma_{\mathrm{rr}}\left(\mathrm{R}_{1}\right)=\sigma_{\mathrm{rr}}\left(\mathrm{R}_{2}\right)=0$.

- b) $\sigma_{\mathrm{rr}}\left(\mathrm{R}_{1}\right)=\sigma_{\mathrm{rr}}\left(\mathrm{R}_{2}\right)=\varepsilon_{\mathrm{zz}}(\mathrm{r})^{1}=0$.

- c) $\sigma_{\mathrm{rr}}\left(\mathrm{R}_{1}\right)=\mathrm{u}\left(\mathrm{R}_{2}\right)=0$.

- d) $\sigma_{\mathrm{rr}}\left(\mathrm{R}_{1}\right)=\mathrm{u}\left(\mathrm{R}_{2}\right)=\varepsilon_{\mathrm{zz}}(\mathrm{r})=0$.

- e) $u\left(R_{1}\right)=\sigma_{\text {rr }}\left(R_{2}\right)=0$.

\footnotetext{
${ }^{1}$ This is true because we suppose that the load is radial only and uniform along the $z$ axis.
} 
Fig. 8 shows the results of the stress distribution for each constraint hypothesis. Comparing the results, it is interesting to notice how constraints modify the stress distribution. Specifically, with respect to the unconstrained solenoid, the circumferential stress is lower and the radial stress behavior also changes, but for constrain condition b), where there is a increase of the axial stress due to the axial constrain that forbids axial solenoid deformations.
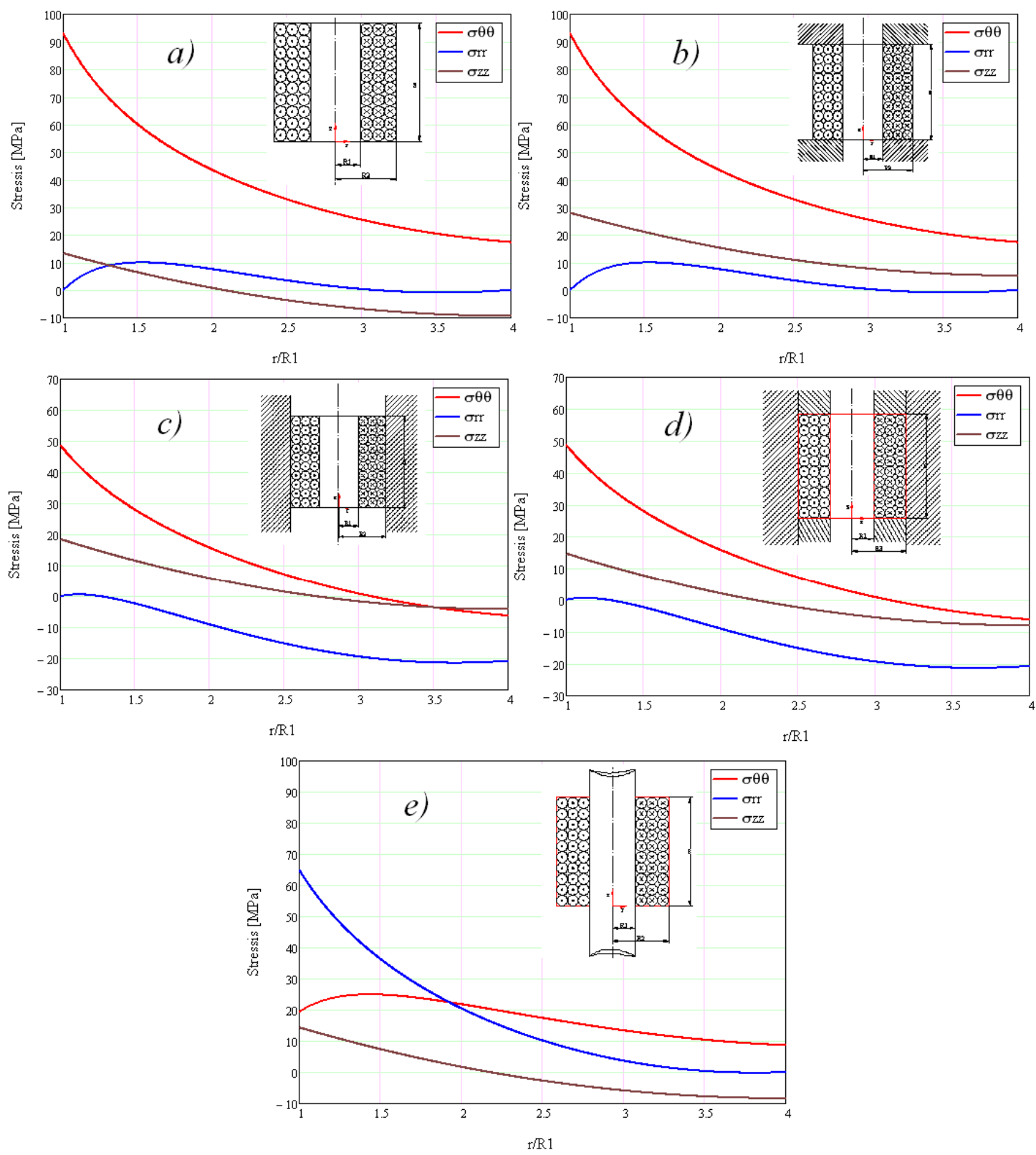

Fig. 8. Stress results for each constraint hypotheses. 


\subsection{Stress state as a function of Self-Field}

Observing the stress results of Fig. 8, one notices that on the inner surface the hoop stress is prevalent with respect to the radial and axial stress but for constrain condition $e$ ), where the radial stress is prevalent. Certainly we can say that the critical location (where stresses are highest) of the coil is on the inner surface $(r=R l)$.

The hoop stress (and radial stress for condition e)) at the critical location was calculated as a function of the coil magnetic field, calculated with equation (1), and therefore of size, for constant current density. This is shown in Fig. 9.
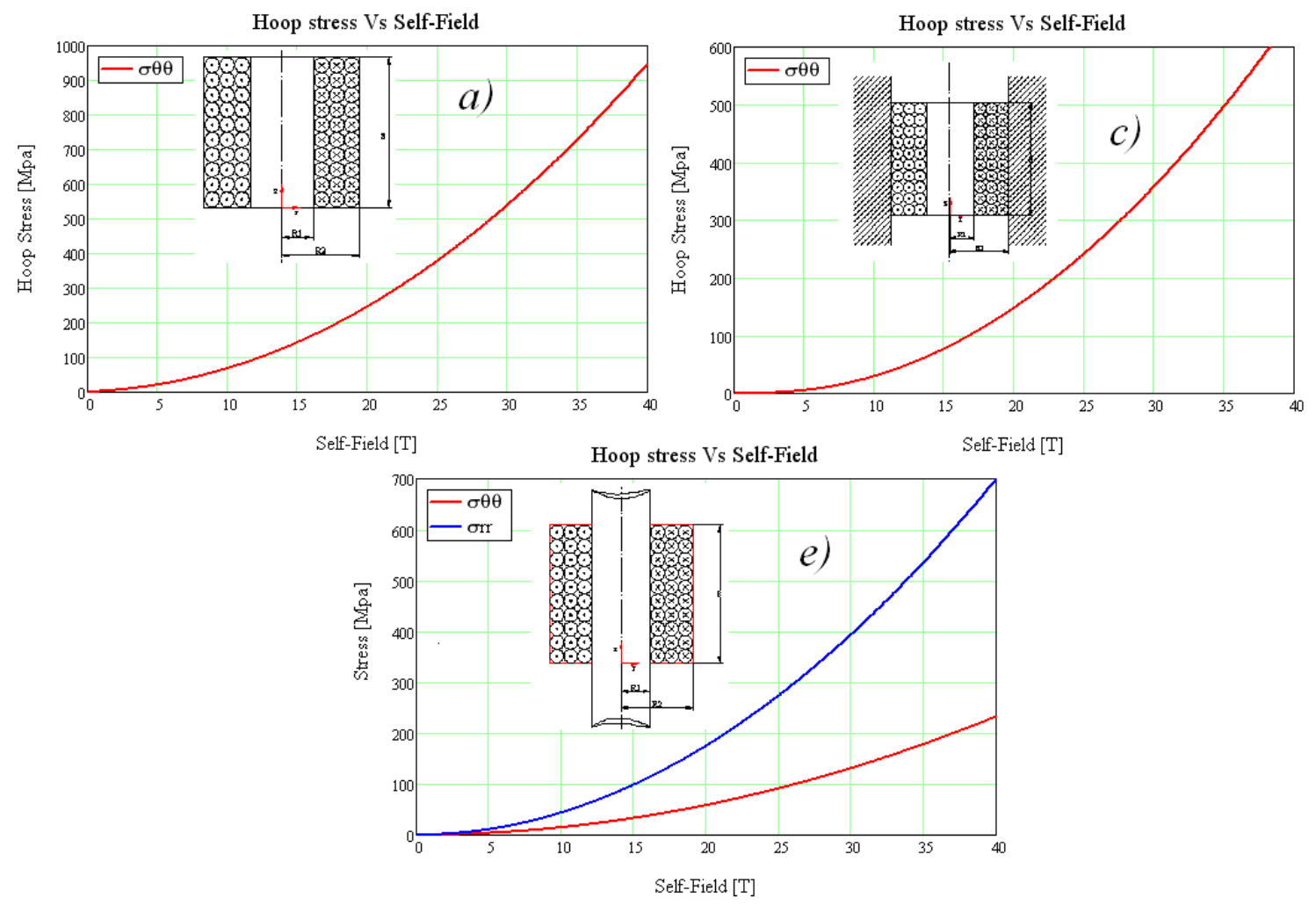

Fig. 9. Stresses vs. Self-Field.

The hypothesis of a constraint on the outer surface is the most realistic case, as all solenoids have an outer skin (generally made of stainless steel) with the purpose of reducing hoop strains. For condition $c$ ), the hoop stress was calculated for several values of the current density; as shown in Fig. 10. 




Fig. 10. Hoop stress vs. Self-Field for a infinite length solenoid and several values of the current density. 


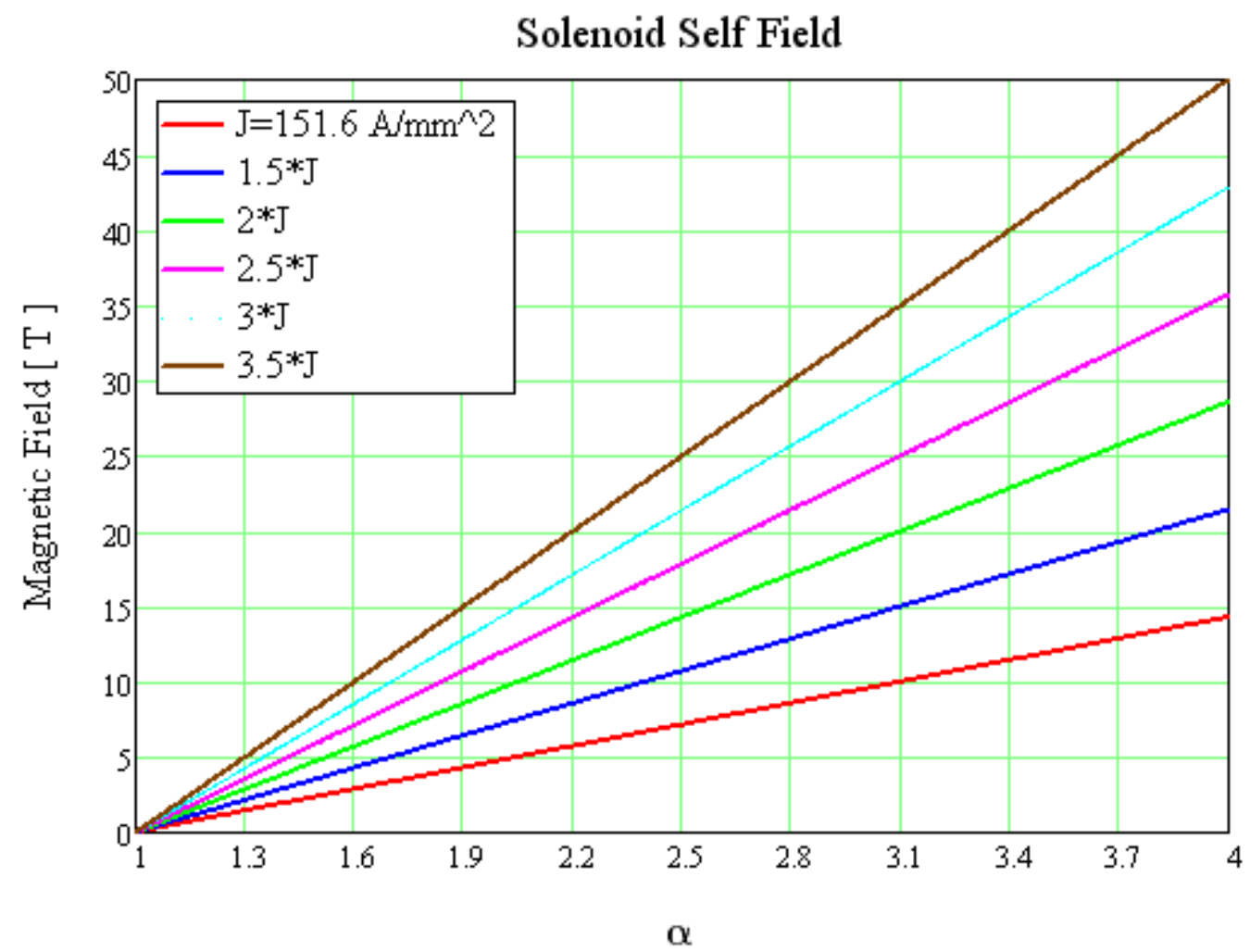

Fig. 11. Self-field vs. a for an infinite length solenoid and several values of the current density.

The above plot shows that given a field value, as we increase current density, the resulting solenoid is smaller (see Fig. 11) and produces a larger hoop stress.

In the following, the comparisons of the analytical results with the FEM results are shown for the more realistic case $c$ ).

\section{Analytical model vs. FEM}

To validate the analytical solution, a comparison with FEM has been made for the same geometry as in Section 2.1 in the case of the $c$ ) hypothesis. The FEM model was run using the same assumptions of the analytical model, namely infinite rigidity of the constraints and room temperature operation. 
The first comparison was made on the magnetic field results (see Fig. 12), where we can see that the FEM solution shows a non uniform field in the inner bore of the coil and a different value from zero at the outer surface $(r=R 2)$.

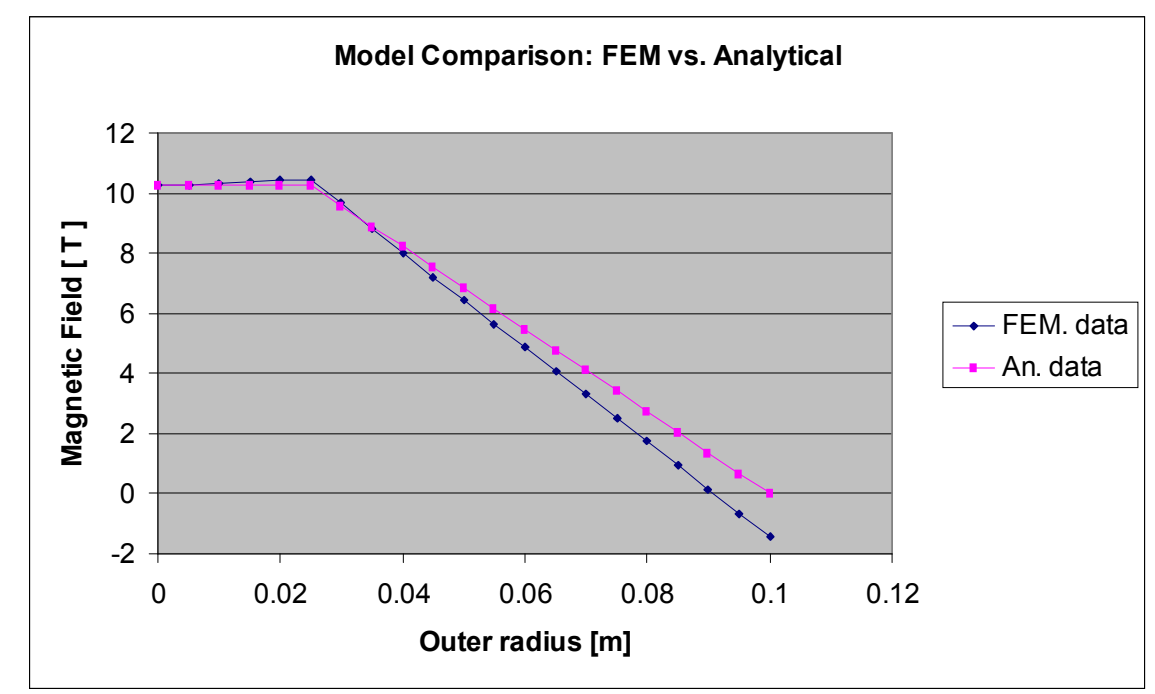

Fig. 12. Magnetic Field vs. radial coordinate at the middle section $z=L / 2$.

The comparisons for the hoop and radial stresses are shown in Fig. 13. 

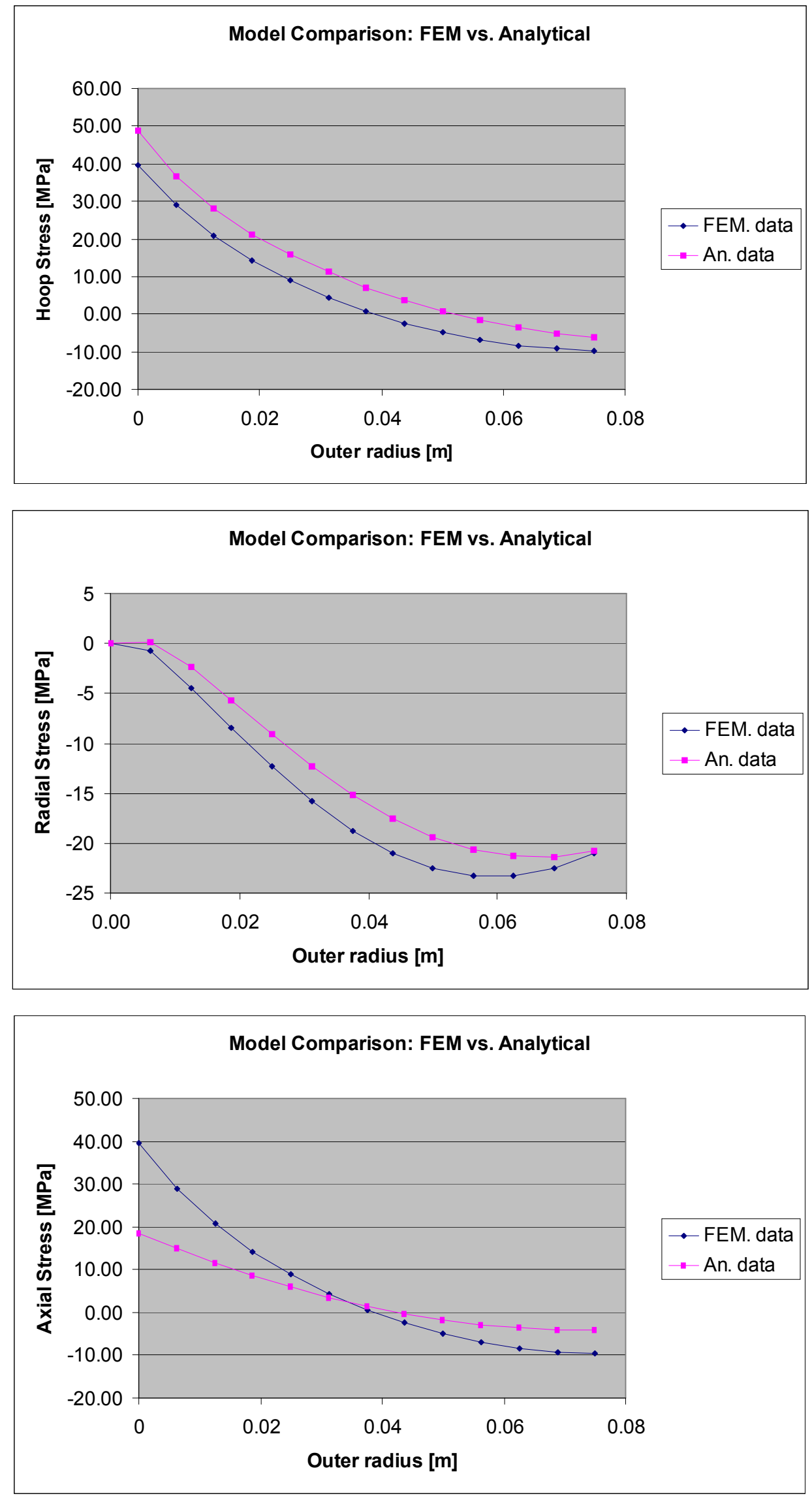

Fig. 13. Stress Results comparison. 
From the analysis of Fig. 13 we note immediately that whereas the analytical hoop and radial stresses are close to the FEM results, there is a large difference in the axial stresses. The reason can be found in the fact that in the analytical model, the radial component of the magnetic field that gives an axial load has been neglected. This is true in the middle section of the solenoid $(z=L / 2)$ and the effect is as much stronger the longer the solenoid.

Fig. 14 shows a map of radial components of the magnetic field in one half of the cross section of the solenoid, where it can be seen that the largest radial components are at the top and bottom ends.

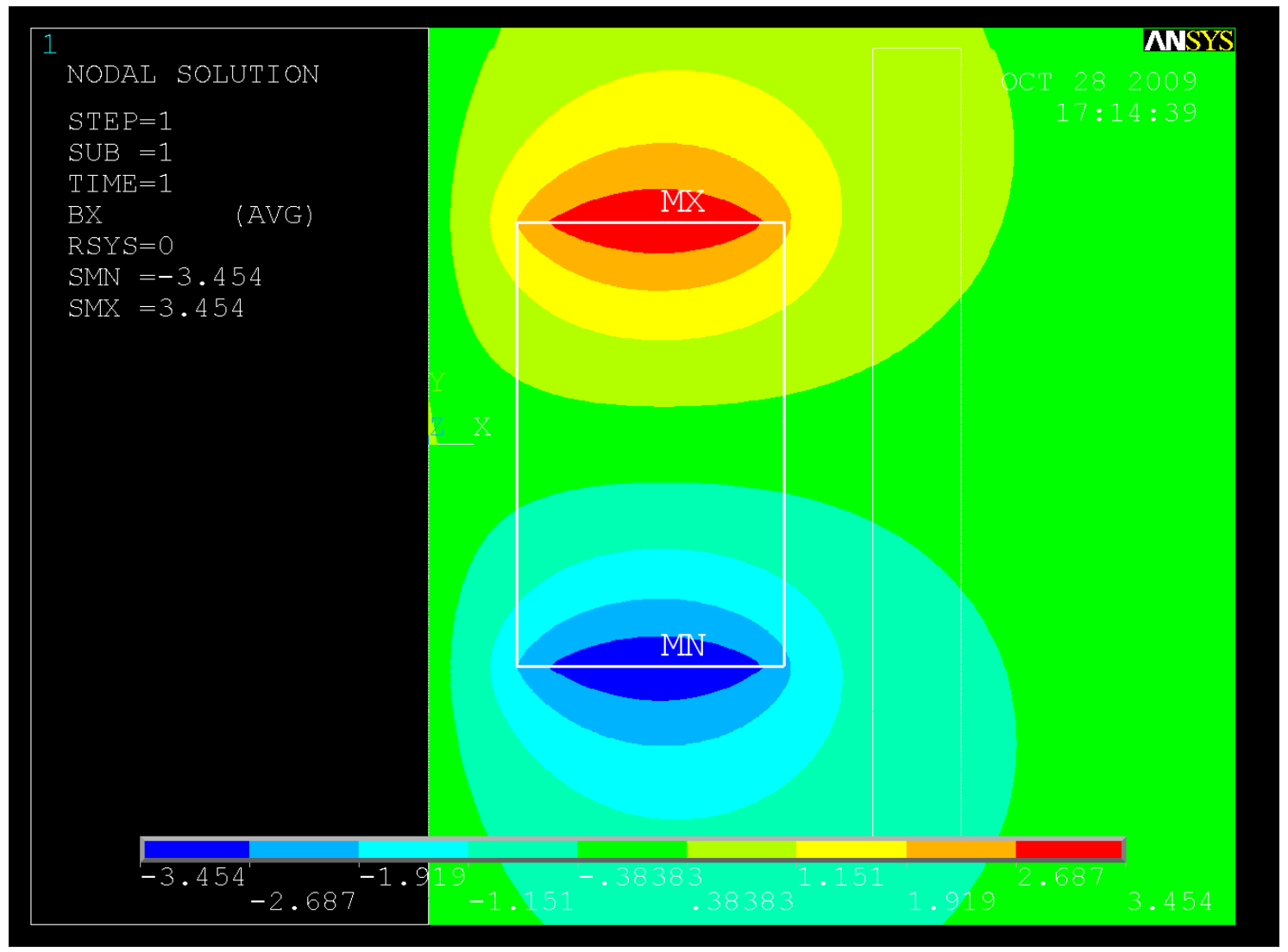

Fig. 14. Map of radial components of magnetic field in one half of the cross section of the solenoid. 


\section{Effect of temperature and of finite rigidity of the constraints (BSCCO vs. YBCO coils)}

Finite element modeling was used also to check the following effects for constraint hypothesis $c$ ). One effect was that of using an infinite stiffness for the outer constraint as opposed to a stainless steel skin with a Young's modulus equal to $206^{*} 10^{9} \mathrm{~Pa}$ and a thickness of $2 \mathrm{~mm}$. In the latter case, the Young modulus used for BSCCO was $E$ $=40 * 10^{9} \mathrm{~N} / \mathrm{m}^{2}$, a value deemed representative of a brittle and epoxy impregnated conductor [6]. For YBCO, $E=110^{*} 10^{9} \mathrm{~N} / \mathrm{m}^{2}$. The comparisons of the stresses obtained with these three FEM models are shown in Fig. 15.

The thermal effects at $4.2 \mathrm{~K}$ as opposed to a room temperature model were calculated separately for BSCCO and for YBCO using the same Young moduli as above. The comparisons of the stresses obtained with these FEM models are shown in Fig. 16 for BSCCO and in Fig. 17 for YBCO. In all cases a glued connection was used between solenoid and skin. Such hypothesis has the most impact on the radial stress. 

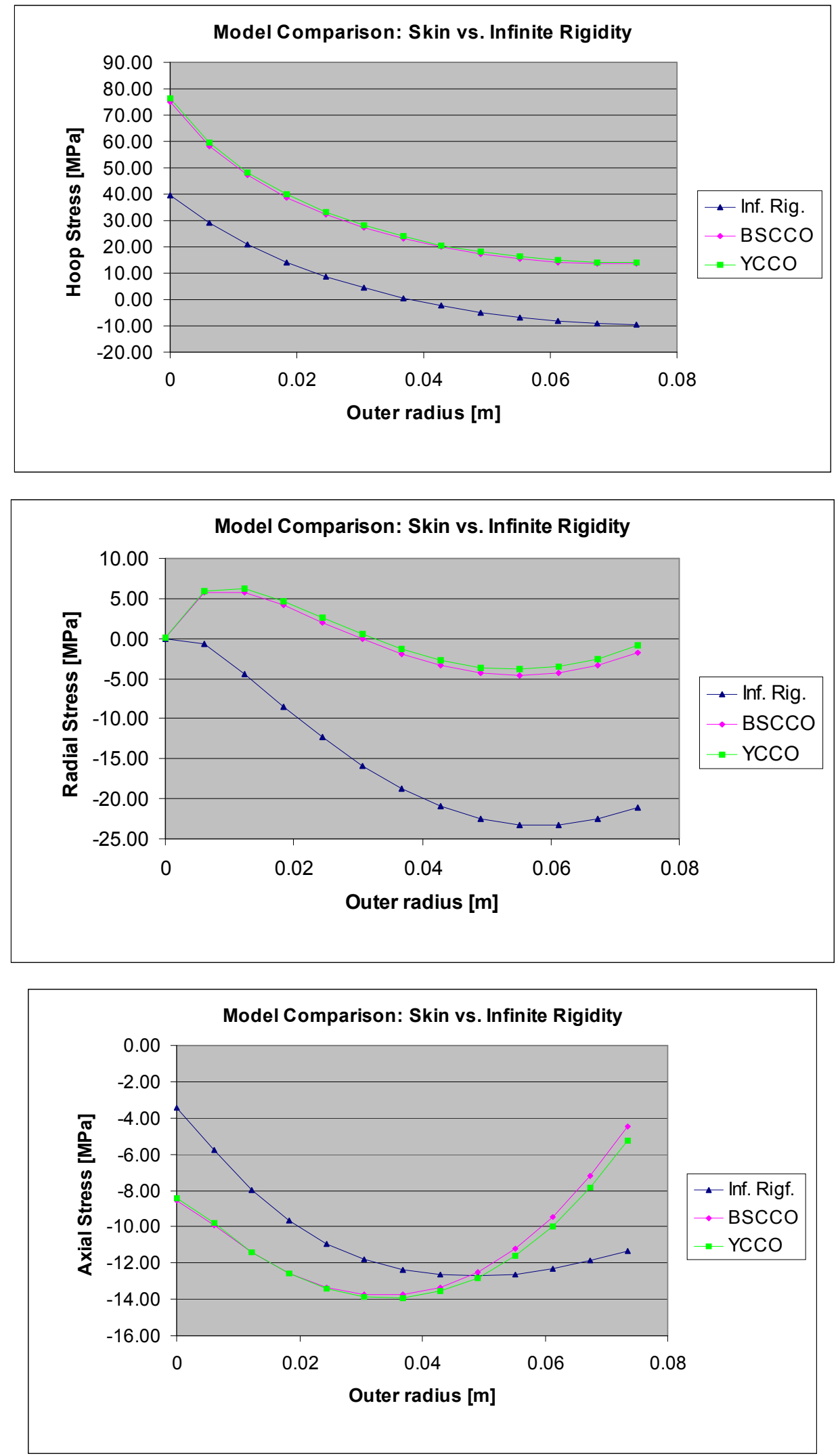

Fig. 15. Comparison of the FEM results for BSCCO and YBCO vs. case with infinite rigidity. 

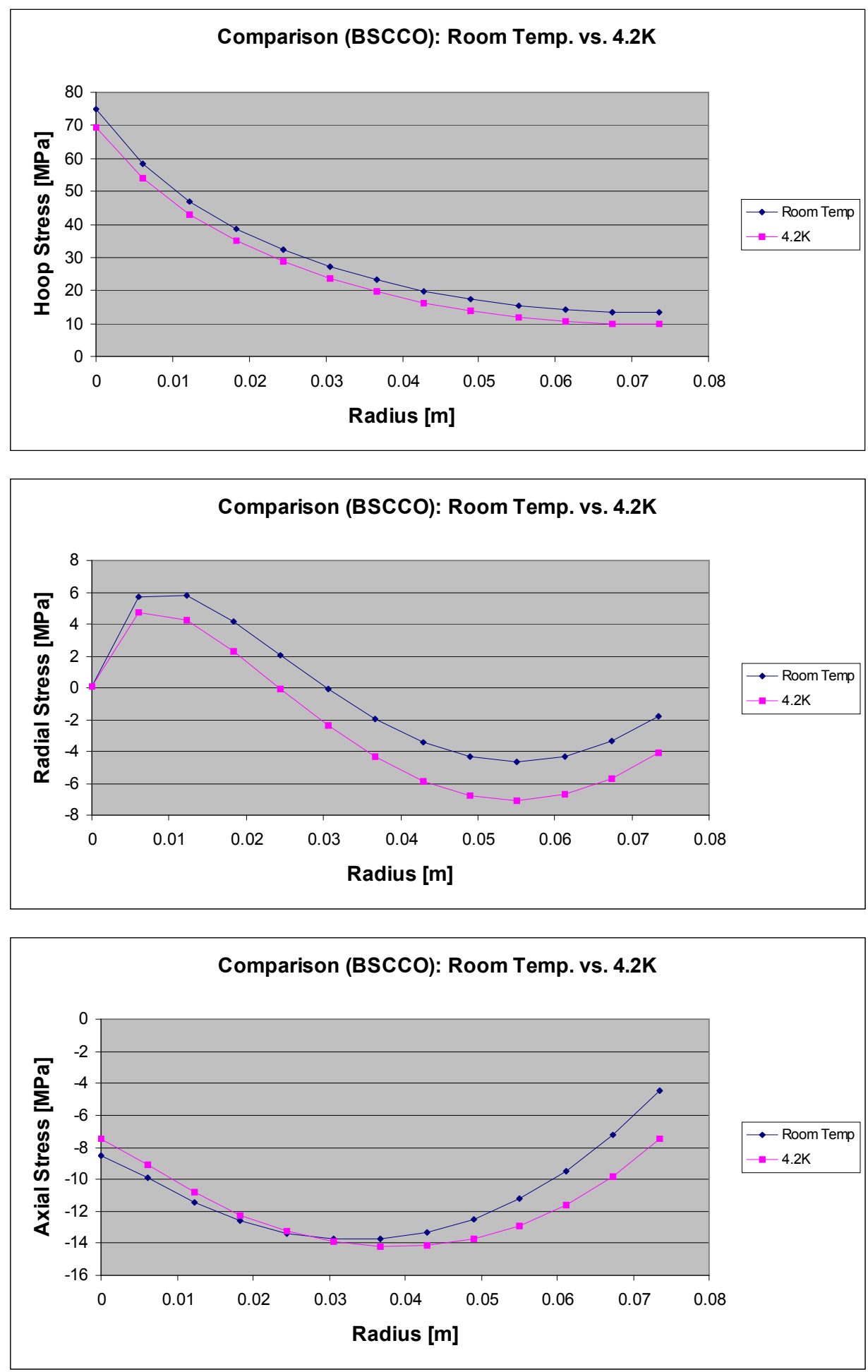

Fig. 16. Comparison of the FEM results for BSCCO at room temperature vs. $4.2 \mathrm{~K}$. 

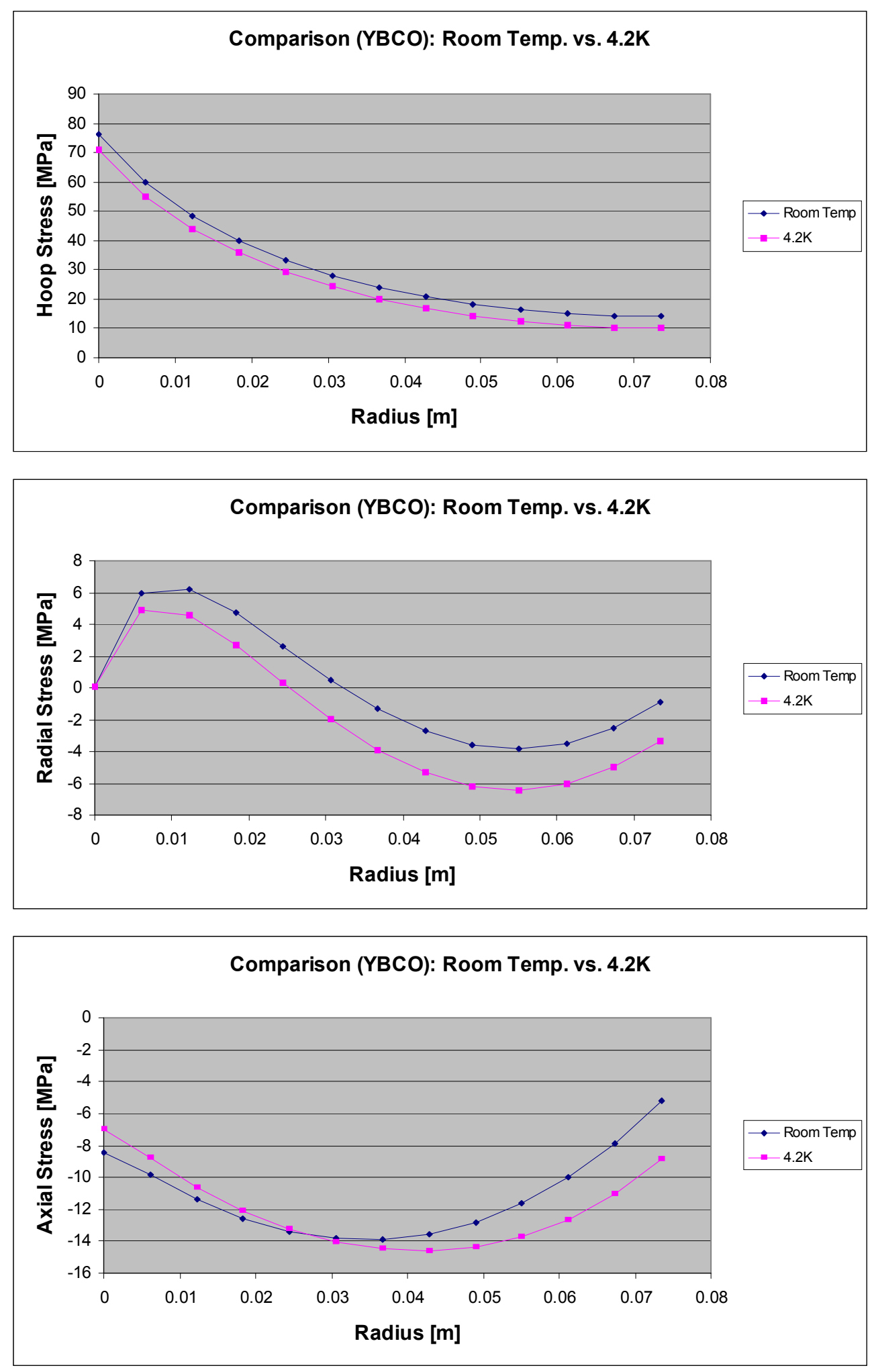

Fig. 17. Comparison of the FEM results for YBCO at room temperature vs. $4.2 \mathrm{~K}$. 


\section{Conclusion}

Using an analytical model a preliminary stress study of high field solenoid was performed for several constraints hypotheses. It was shown that the constraints modify the stress behavior. Specifically, by constraining the solenoid on its outer surface, the hoop stress decreases in the coil. A comparison of the analytical results and FEM results was performed to check how closely the analytical model reproduced the FEM model. Whereas the analytical model predicts quite well the radial and azimuthal stresses, the largest differences were found in the axial stresses. Not including the thermal effects at 4.2 $\mathrm{K}$ makes the analytical model conservative, as the FEM models comparing room temperature to $4.2 \mathrm{~K}$ operation show that the stresses at $4.2 \mathrm{~K}$ are lower. The analytical model is however a good tool for conceptual design. To deepen the understanding of the mechanical aspects in these coils, these studies will be continued using finite element models.

\section{References}

[1]D. Turrioni et al., "Study of HTS Wires at High Magnetic Fields", accepted for publication in IEEE Trans. Appl. Sup., Special Issue (2009).

[2] Halliday, Resnick, Walker, "Fundamentals of Physics"

[3] Martin N. Wilson, "Superconducting Magnets", Oxford Science Publications, 1997.

[4] R.W. Boom, R.S. Livingston, "Superconducting Solenoids", Proceedings of the IRE, March 1961.

[5] Timoshenko S., "Strength of materials", Princenton, New Jersey, 1956.

[6]D.R. Chichili et al, "Investigation of cable insulation and thermo-mechanical properties of epoxy impregnated $\mathrm{Nb}_{3} \mathrm{Sn}$ composite", IEEE Trans. Appl. Sup., Vol. 10, No. 1, pp. 1317-1320, 2000. 